MATEC Web of Conferences 25, 03018

(2015)

DOI: $10.1051 /$ matecconf/ 20152503018

(C) Owned by the authors, published by EDP Sciences, 2015

\title{
An Adaptive Color Image Visible Watermark Algorithm Supporting for Interested Area and its Application System Based on Internet
}

\author{
Guoqing Hu \& Xinlong Chen \\ College of Communication Engineering, Chongqing University, Chongqing, China
}

\begin{abstract}
This paper presents an interested area visible watermark algorithm for digital color image based on internet. By converting color image from RGB to HSI and using the original image information to obtain the watermark coefficient, the algorithm embeds watermark on the spatial domain and selects the interested area by clicking on the picture. By visiting the site (http://dgdz.ccee.cqu.edu.cn/watermark/watermarkb.aspx) and uploading the original image and watermark image, the color image with the visible watermark can be gained on web. Experimental results show that the algorithm has only a little influence on the original image and the image of watermark is clearly visible. It is very suitable for obvious identification of the copyright from digital image through Internet.
\end{abstract}

Keywords: visible watermark; color image; Internet

\section{INTRODUCTION}

In the era of knowledge, the intellectual property right, which is the concentrated expression of the core competitiveness of enterprises, is the important wealth and resource of factors in production. Nowadays, more and more problems of intellectual property rights infringement are generated by digital image transmission. The copyright protection and content authentication method and its application system for digital image that based on the Internet have important significance. Digital image watermarking technology, a powerful tool for digital image copyright protection and content authentication, has been widely concerned by scholars.

There are many research results in digital image watermark technology, including visible and invisible watermarks. The invisible watermark requires that the embedded watermark should be not only transparent to observers ${ }^{[1]}$, but also robust enough so that it cannot be easily destroyed or removed after some digital image processing or attacks ${ }^{[2]}$. According to the watermark embedding technology, the digital watermark is divided into spatial domain and transform domain ${ }^{[3]}$. The existing frequency transformation methods for watermark embedding include discrete Fourier transform (DFT) ${ }^{[4]}$, discrete cosine transform $(\mathrm{DCT})^{[5]}$, and discrete wavelet transform (DWT) ${ }^{[6]}$. In recent years, watermarking techniques have been improved using optimization algorithms such as genetic algorithm (GA) which is a popular evolutionary optimization technique invented by Holland ${ }^{[7]}$. In the field of watermarking, GA is mainly used in the embedding procedure to search for locations to embed the watermark ${ }^{[8-11]}$.

The visible watermark can be seen to the observer and is particularly suitable for the identification of copyright. It is often used to prevent or stop the illegal use of high quality image, which is protected by copyright. Some specific watermark information has advertising effect. In order to better promote or sell their works, many media makers embedded the visible watermark information into the pictures before the pictures posted to the Internet and designing a color image visible watermark scheme and its application system, based on internet, are of great significance to the further promotion of digital watermark technology.

\section{ALGORITHM BASED ON SPATIAL DOMAIN}

With the further deepening of the application for the digital library, the study on the visible watermark is gradually attracting attention. Some visible watermark algorithms are as follows:

The visible watermark algorithm based on spatial domain, the visible watermark algorithm based on discrete cosine transform (DCT) and the visible watermarking algorithm based on discrete wavelet transform domain and so on.

The algorithm, based on spatial domain, is relatively simple. It gains the original watermark embedding coefficient by the original image details of each area and has a little influence on the low resolution sub blocks of the original image.

The algorithm based on transform domain is more complex. For example, the algorithm based on DCT domain transforms the image from the spatial conversion to DCT domain, then calculates the watermark embedding factor according to the image content features, and uses IDCT to transform the image to spatial domain.

Considering the need of internet application, this paper presents a visible watermark algorithm for digi- 


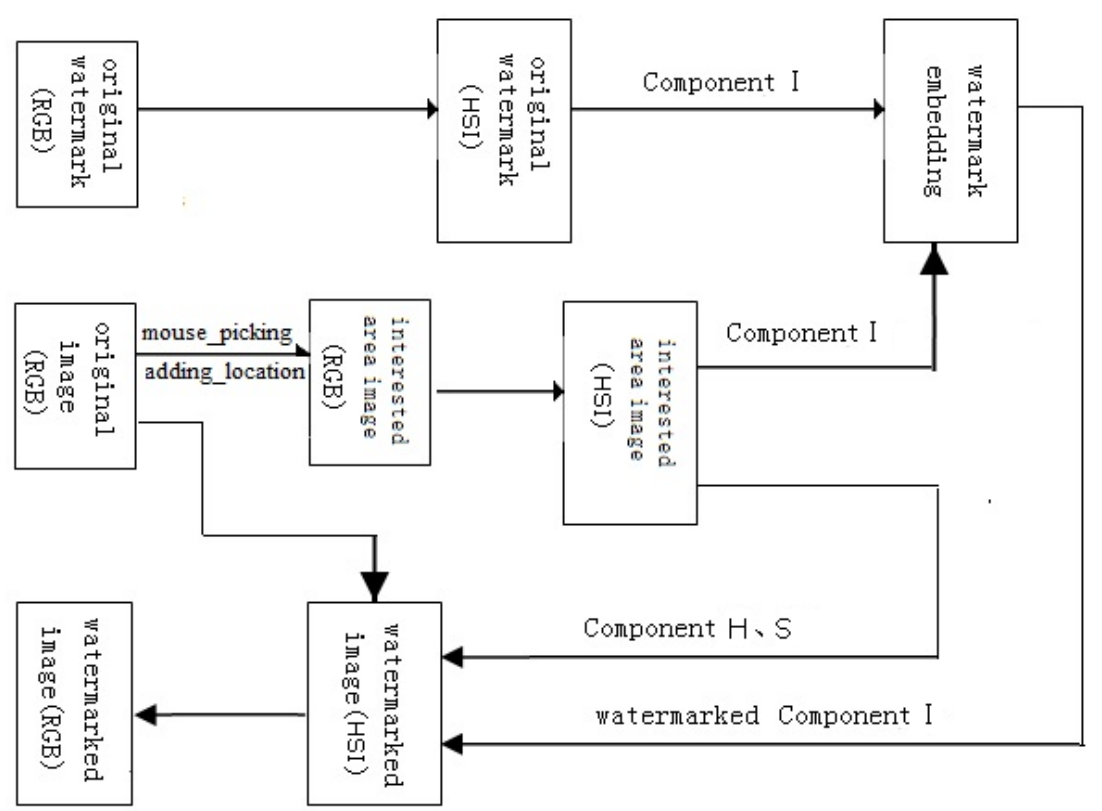

Figure 1. Algorithm of watermark embedding

tal color image based on spatial domain and supported for interested area.

\section{WATERMARK EMBEDDING}

The algorithm of watermark embedding is shown in Figure 1, explained briefly as Figure 1.

First, the algorithm uses the FileUpload component of .NET to obtain the files of the original image and the original watermark, and uses .NET framework GDI+ technique to obtain the data of images. When the width of original image or original watermark has more than 800 pixels, or the height has more than 600 pixels, the application system will adjusts the image with 800 pixels width and 600 pixels height automatically.

Follows the steps of using .NET framework GDI+ technique to obtain the image and finish the processing of the image.

Using the bitmap class of the.NET GDI+ to object a new instance of the Bitmap object box, the Width, Height and other attribute information of the original image and original watermark will be obtained. Creating a new instance of the Color object, then you can gain the original image pixels and the original watermark image pixels of RGB component.

Second, the algorithm uses the ImageClickEventArgs function of .NET to obtain the specific location of the original image, which is clicked by the mouse and is the starting location of watermark embedding.

After that, the RGB images of the original image and the original watermark are converted to HSI space.
HSI model is the most commonly-used model for color processing, explained briefly as follows:

H: hue

S: saturation

I: density (corresponding to luminance and image gray).

The relationship from RGB color space to HIS color space is as follows:

$$
\left\{\begin{array}{l}
I=\frac{1}{3}(R+G+B) \\
S=1-\frac{3}{(R+G+B)}[\min (R+G+B)] \\
H=\arccos \left\{\frac{[(R-G)+(R-B)] / 2}{\left[(R-G)^{2}+(R-B)(G-B)\right]^{1 / 2}}\right\}
\end{array}\right.
$$

Then, the embedded area is determined in the original image and the embedding area size is as same as the size of the watermark image.

The data of component I, extracted from the original image and the watermark image, are divided into many $8 * 8$ sub blocks, calculated the average value for the original image, calculated the variance, minimum and maximum and calculated the embedding coefficients of each sub block.

The calculation formula of the embedding coefficients for each sub block is as follows:

$$
F_{\mathrm{ij}}=\frac{\alpha_{\mathrm{ij}}-\alpha_{\mathrm{min}}}{\alpha_{\text {max }}-\alpha_{\text {min }}}
$$

$F_{\mathrm{ij}}$ : embedding coefficient of sub block $(\mathrm{i}, \mathrm{j})$ 


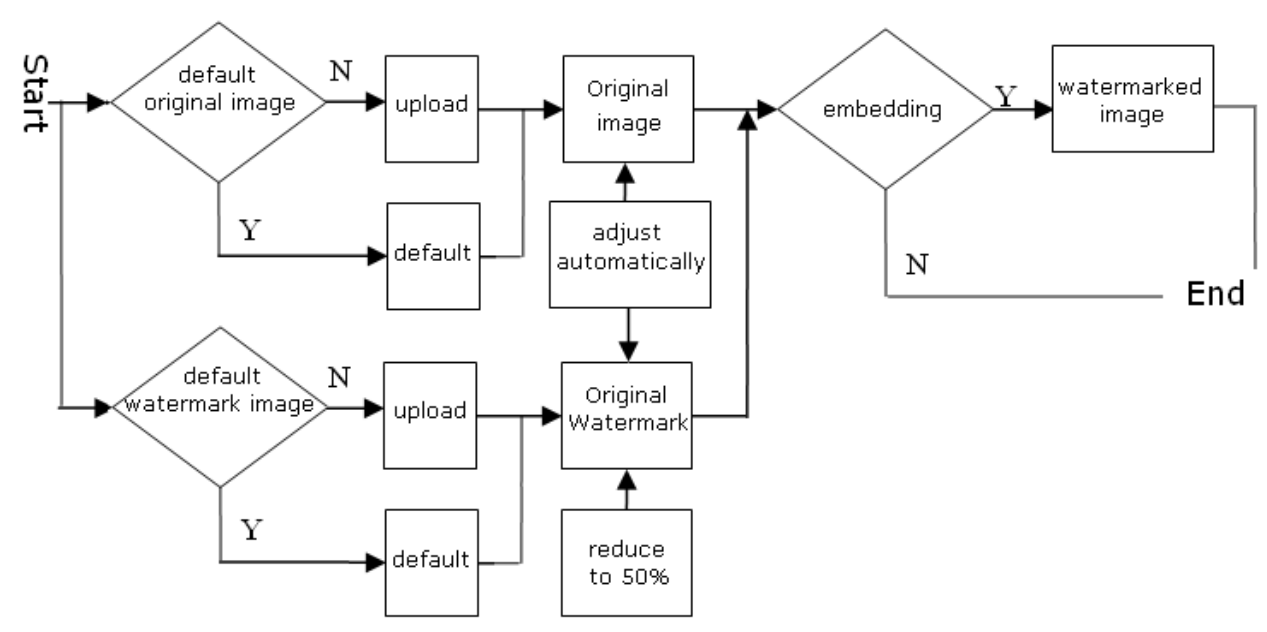

Figure 2. Flow chart of software implementation

$\alpha_{\mathrm{ij}}$ : Variance of sub block (i, j)

$\alpha_{\min }$ : Minimum variance of original image

$\alpha_{\max }$ : Maximum variance of original image

The formula of watermark embedding is as follows:

$I_{1 i j}^{\prime}=F_{m n} I_{1 i j}+\left(1-F_{m n}\right) I_{1 i j} I_{2 i j}$

$F_{\text {mn }}$ : embedding coefficient of sub block $(\mathrm{m}, \mathrm{n})$

$I_{1 \mathrm{i} j}$ : pixel values of position $(i, j)$ from the sub block $(\mathrm{m}, \mathrm{n})$ of original image component I

$I_{2 \mathrm{i} j}$ : pixel values of position $(i, j)$ from the sub block $(m, n)$ of original watermark component I

The formula does point to that, when the value of $I_{2 \mathrm{i} \text { jequals }} 1$ or the value of $F_{\text {mn }}$ equals 1 , the gray value of the original image is not changed. When the value of $F_{\mathrm{mn}}$ is reaching to 1 , the gray value of the watermarked image is very close to the original image and has little influence on the gray value of the original image. Thus the adaptive color image visible watermark algorithm only has influences on the original gray image in low sub block and has little influences on the original gray image in high detail block.

Finally, using I component, $\mathrm{H}$ components and $\mathrm{S}$ components, and converted them to RGB space, then the visible watermarked image can be gained.

When the value of $\mathrm{H}$ is between 0 and 120 , the relationship from HIS color space to RGB color space is as follows:

$$
\left\{\begin{array}{l}
B=I(1-S) \\
R=I\left[1+\frac{S \cos H}{\cos \left(60^{\circ}-H\right)}\right] \\
G=3 I-(B+G)
\end{array}\right.
$$

When the value of $\mathrm{H}$ is between 120 and 240 , the relationship from HIS color space to RGB color space is as follows:

$\left\{\begin{array}{l}R=I(1-S) \\ G=I\left[1+\frac{S \cos \left(H-120^{\circ}\right)}{\cos \left(60^{\circ}-H\right)}\right] \\ B=3 I-(R+G)\end{array}\right.$

When the value of $\mathrm{H}$ is between 240 and 360 , the relationship from HIS color space to RGB color space is as follows:

$\left\{\begin{array}{l}G=I(1-S) \\ B=I\left[1+\frac{S \cos \left(H-240^{\circ}\right)}{\cos \left(300^{0}-H\right)}\right] \\ R=3 I-(G+B)\end{array}\right.$

\section{APPLICATION SYSTEM BASED ON INTER- NET}

The flow chart of software implementation is shown in Figure 2.

The test site is as follows:

http://dgdz.ccee.cqu.edu.cn/watermark/watermarkb. $\underline{\operatorname{aspx}}$

The first interface from the website of the application system is as shown in Figure 3.

The steps of watermark embedding are as follows:

\subsection{Upload original image}

When you have clicked the Browse button and selected the file of original image from the PC, you can click the Upload button to upload the file to the server. If only for testing purposes, you can click the default button directly to get the standard Lena image. When 
MATEC Web of Conferences

The Color Image Visible Watermark Application System

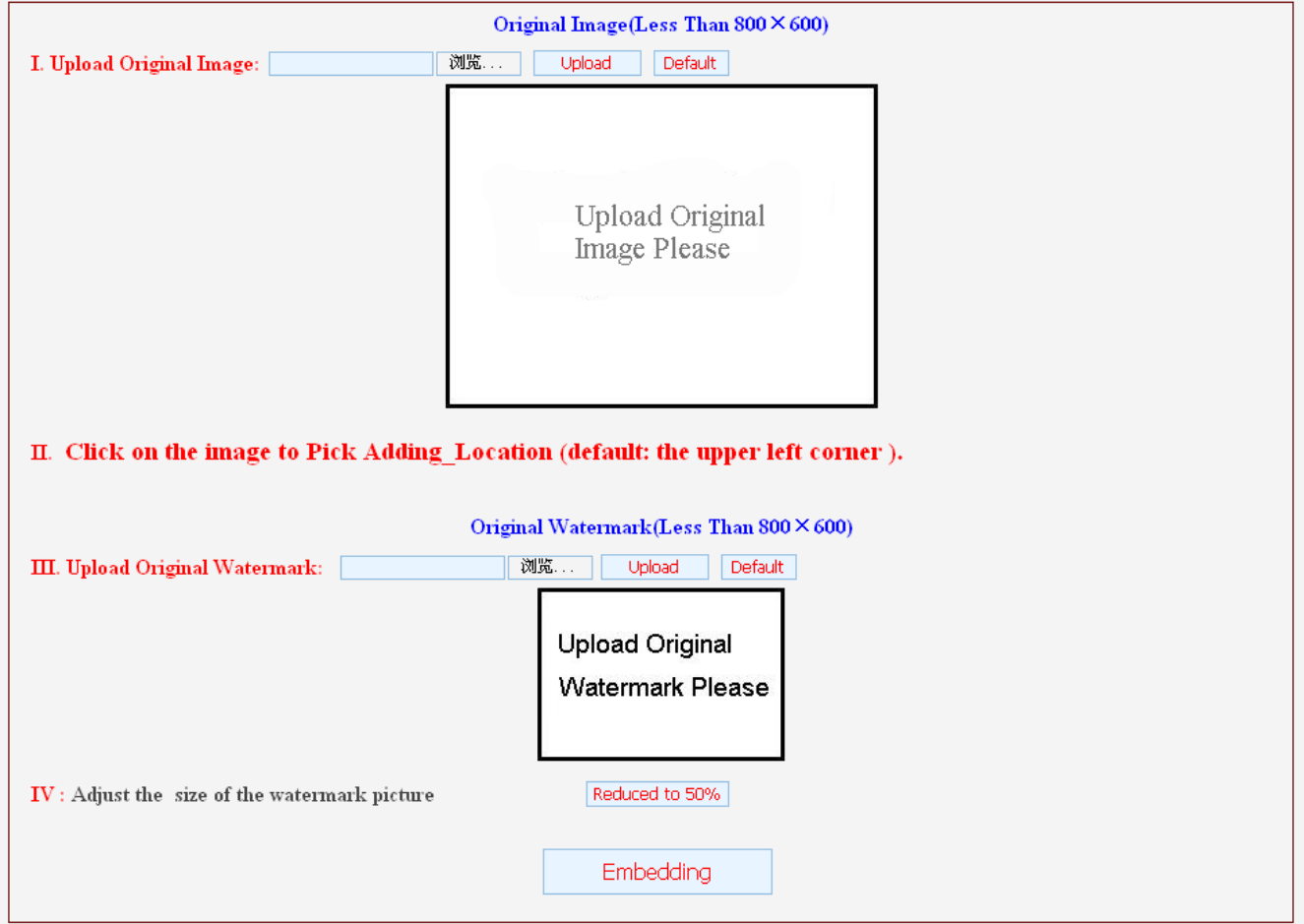

Figure 3. The first interface from the website

the width of original image has more than 800 pixels, or the height has more than 600 pixels, the system will adjust the image with 800 pixels width and 600 pixels height automatically.

\subsection{Pick adding location}

The system supports for adding a visible watermark to the interested area of the original image, so you can determine the starting position of the interested area by clicking on a specific location of the original image. The specific values of the starting position that you have selected will be shown below the images in the web page.

\subsection{Upload original watermark}

You can also click the Browse button and selected the file of original watermark from the PC and click the Upload button to upload the file to the server. If only for testing purposes, you can directly click the default button to get the standard original watermark. If the width of original watermark has more than 800 pixels, or the height has more than 600 pixels, the system will adjust the image with 800 pixels width and 600 pixels height automatically.

\subsection{Adjust the size of the watermark picture}

The interested area of the original image will be determined by selecting the starting position of the interested area and the size of the original watermark. The size of the original watermark can be reduced by clicking the Reduce to $50 \%$ button.

\subsection{Watermark embedding}

Finally, you can click on the embedding button to add the watermark, and the watermarked image will be shown in the location of the original image.

\section{RESULTS AND CONCLUSIONS}

When you have uploaded the original image and watermark image to the server, you can click the embedding button to add a watermark into the original image. You can also click the Reduce to $50 \%$ button to reduce the size of the original watermark.

You can click the default button to get the default original image (Figure 4a: the standard color image of Lena) and the default watermark image (Figure 4b: the LOGO of Chongqing University). The watermarked image as shown in Figure 5a will be gained by clicking the embedding button. 
EMME 2015

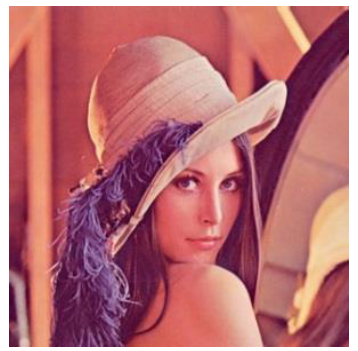

(a) Original image

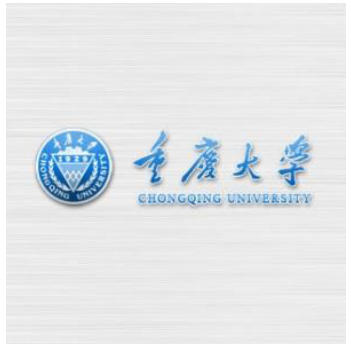

(b) watermark 1

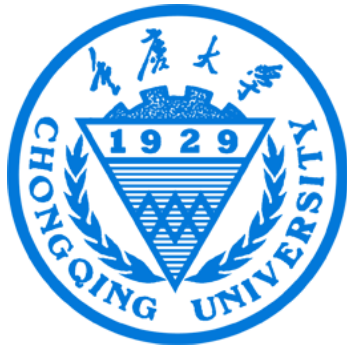

(c) watermark 2

Figure 4. Experimental images based on Lena image

Uploading another watermark image to the server (Figure 4c: another LOGO of Chongqing University), clicking the original image on the position of $(180,180)$ and the embedding button, you can gain the watermarked image (Figure 5b).

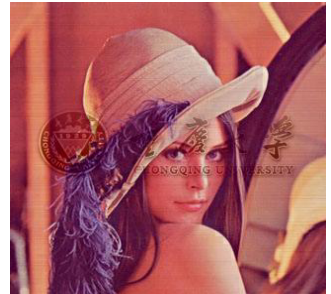

(a) Watermarked image 1

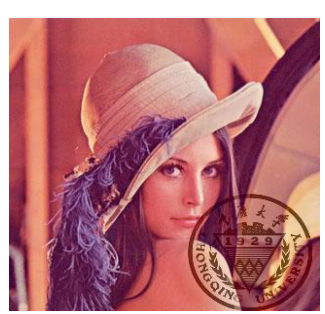

(b) watermarked image 2
Figure 5. Watermarked images based on Lena image

Another test data is shown in Figure 6. The original image is the LOGO of Bank of Ireland (Figure 6a) and the watermark image is another LOGO of Chongqing University (Figure 4c).

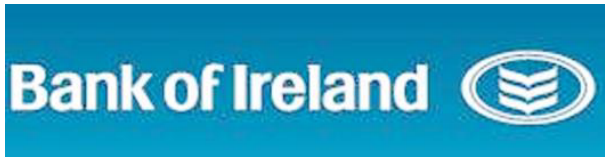

(a) Original image

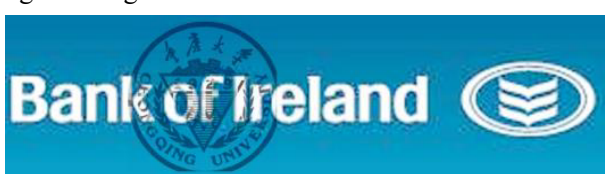

(b) Watermarked image 1

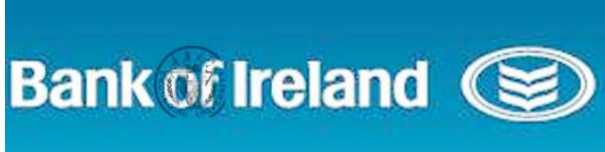

(c) Watermarked image 2

Figure 6. Watermarked images based on the LOGO of Bank of Ireland
Uploading the LOGO of Bank of Ireland and the LOGO of Chongqing University to the server, and clicking the original image on the position of $(158,13)$, then the watermarked image as shown in Figure $6 \mathrm{~b}$ will be obtained.

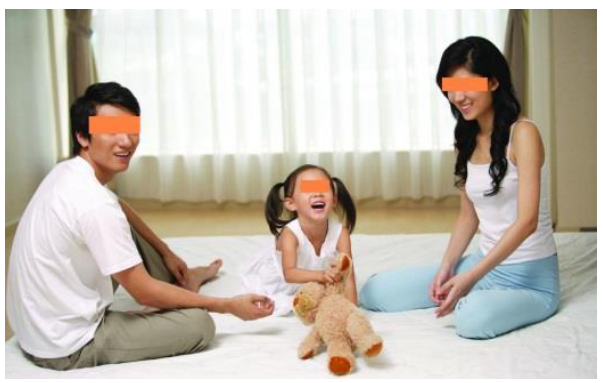

(a) Original image

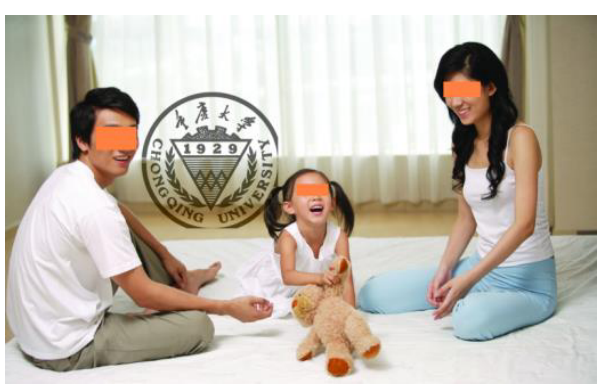

(b) Watermarked image 1

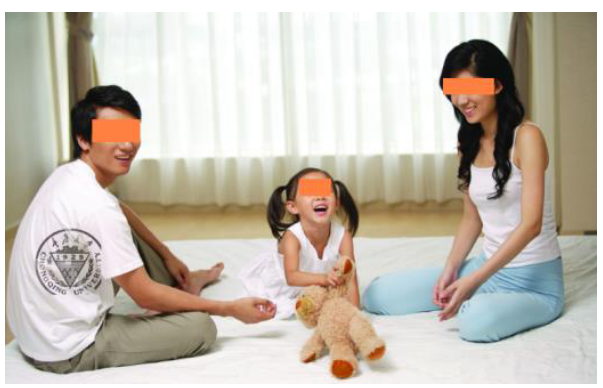

(c) Watermarked image 2

Figure 7. Watermarked images based on the picture of daily life 


\section{MATEC Web of Conferences}

Clicking the original image on the position of (188, 63 ), and clicking the Reduce to $50 \%$ button to reduce the size of the watermark image and the embedding button, you can get the watermarked image (Figure $6 c)$.

The image (Figure 5) shows that the LOGO of Chongqing University is clearly visible, and the image (Figures $6 \mathrm{~b}, 6 \mathrm{c}$ ) shows that both the LOGO of Bank of Ireland and the LOGO of Chongqing University are clearly visible.

This method is also suitable for adding visible watermark to the image of daily life, and the test data are shown in Figure 7. The original image is a photo of a family of three; the watermark image is the LOGO of Chongqing University (Figure 4c).

Uploading the original image and the watermark image to the server, and clicking the original image on the position of $(285,133)$, the watermarked image as shown in Figure $7 \mathrm{~b}$ will be obtained.

Clicking the original image on the position of (358, 43 ), and clicking the Reduce to $50 \%$ button to reduce the size of the watermark image and the embedding button, you can get the watermarked image (Figure $7 \mathrm{c})$

Through the test data, it is not difficult to get the conclusions as follows:

The adaptive color Image visible watermark algorithm, supporting for interested area, has only a little influence on the original image, and the image of watermark is clearly visible and provides a good protection for original image. Because the impact of the visible watermark on the color image quality is slight and both are clearly visible, it is very suitable for obvious identification of the copyright from digital image through Internet.

\section{REFERENCES}

[1] W. Wang, W.H. Li, Y. k. Liu, \& Z. Borut, 2014. A SVD
SVD Feature based Watermarking Algorithm for Gray-level Image Watermark, Journal of Computers, 9: 1497-1502.

[2] Wei Wang, \& Chengxi Wang. 2008. A Watermarking Algorighm for Gray-level Watermark based on Local Feature Region and SVD, International Congress on Image and Signal Processing, pp.650-654.

[3] R B Wolfgang, C I Podilchuk, \& E J Delp. 2007. Perceptual watermark for digital image and video. Pro IEEE, 87(1):1108-1126.

[4] M. David, S. R. Jordi, \& F. Mehdi. 2010. Efficient selfsynchronised blind audio watermarking system based on time domain and FFT amplitude modification, Signal Processing. 90: 3078-3092.

[5] W. Liu \& C. H. Zhao. 2009. Digital watermarking for volume data based on 3D-DWT and 3D-DCT, The 2nd International Conference on Interaction Sciences: Information Technology, Culture and Human, pp.352-357.

[6] B. Deepayan \& A. Charith. 2010. Video watermarking using motion compensated $2 \mathrm{D}+\mathrm{t}+2 \mathrm{D}$ filtering, The 12th ACM Workshop on Multimedia and Security, pp.127-136

[7] J. Holland, 1975. Adaptation in natural and artificial systems, University of Michigan Press, Ann Arbor, MI

[8] P. Kumsawat, K. Attakitmongcol, \& A. Srikaew. 2005. A new approach for optimization in image watermarking by using genetic algorithms, IEEE Transactions on Signal Processing, 53: 4707-4719.

[9] Y. T. Wu \& F. Y. Shih. 2006. Genetic algorithm based methodology for breaking the steganalytic systems, IEEE Transactions on Systems, Man, and Cybernetics, 36: 24-31.

[10]H. C. Huang, J. S. Pan, Y. H. Huang, F. H. Wang, \& K.C. Huang. 2007. Progressive watermarking techniques using genetic algorithms, Circuits Systems Signal Processing, 26: 671-687.

[11]S. C. Chu, H. C. Huang, Y. Shi, S. Y. Wu, \& C. S. Shieh. 2008. Genetic watermarking for zerotree-based applications, Circuits Systems Signal Process, 27: 171-182. 